same difficulties. A feasible method for solving quadratic equations by modular computation can, however, be based upon the Taylor expansion $(1-4 x)^{-1 / 2}=$ $\sum_{n=0}^{\infty}\left(\begin{array}{c}2 n \\ n\end{array}\right) x^{n}$.

6. Concluding Remarks. Preliminary analysis indicates that parallel computation, using modular arithmetic, is feasible for certain kinds of problems. The parallel computation envisioned here leads very swiftly to a solution encoded "in modular notation." By this is meant, a system of simultaneous congruences, whose solution (in a specified interval), written as a binary number, has as its initial digits the binary number which is the goal of the computation. For results of practical value it will probably be necessary, at the very least, to use moduli whose product exceeds $10^{10}$. Hence the feasibility of rapid solution of large-scale systems of congruences will determine the timesaving possibilities of the method. Any a priori knowledge about the solution, such as might be obtainable from a preliminary rough solution, analog computation, etc., leads to a reduction in the number of necessary moduli, i.e., knowledge of $r$ binary places reduces the product of the $m_{i}$ needed by a factor $2^{-r}$. Again, in such a case as the boundary value problem, where the values of the solution at neighboring net points differ by amounts which can be bounded a priori, this fact might lead to a considerable reduction of labor in the "conversion" phase of the problem.

7. Acknowledgment. The author wishes to thank Lt. Col. L. M. Butsch, Jr., Capt. F. M. Brown, and Capt. A. L. Calton, Jr. of the Bionics and Computer Laboratory, Wright Air Development Division, for directing his attention to the area of modular computation, and Dr. D. L. Slotnick of the Westinghouse Electric Corporation, Air Arm Division, for many stimulating discussions on the subject.

Institute of Mathematical Sciences

New York University

New York 3, N. Y.

\title{
Permutations with Restricted Position
}

\section{By Frank Harary}

In his book on combinatorial analysis, Riordan [4, p. 163-164] discusses permutations with restricted position and mentions an open question:

"Any restrictions of position may be represented on a square, with the elements to be permuted as column heads and the positions as row heads, by putting a cross at a row-column intersection to mark a restriction. For example, for permutations of four (distinct) elements, the arrays of restrictions for the rencontres and reduced ménage problems mentioned above are 


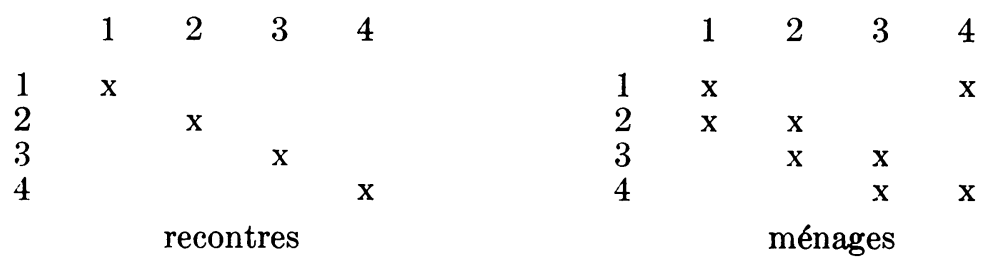

Since a square of side $n$ has $n^{2}$ cells, and a cross may or may not appear in each cell, it is clear that with $n$ elements $2^{n^{2}}$ problems are possible (this includes permutations without restriction, for which no cell has a cross). However, many of these are not distinct since, from the enumeration standpoint, the relative rather than the absolute position of the crosses is important; for example, all $n^{2}$ problems having just one cross on the board are alike. The exact number of distinct problems, for any $n$, is not known, but some progress in this direction will appear in this chapter."

In this note, we show that the question has been virtually solved in [2], and shall obtain an explicit formula for $P_{n}$, "the exact number of distinct problems, for any $n . "$ For we shall see that the chromatically nonisomorphic bicolored graphs with $n$ points of each color, which are enumerated in [2], are in a one-to-one correspondence with the distinct problems involving permutations on $n$ objects with restricted position.

A binary matrix is one in which every entry is $\mathbf{0}$ or 1 . Consider the set $\mathbf{M}$ of all square binary matrices of order $n$. We say that two matrices $A$ and $B$ in $\mathbf{M}$ are equivalent if $B$ can be obtained from $A$ by the following three operations:

1. Perform any permutation on the rows of $A$, obtaining $A_{1}$

2. Perform any permutation on the columns of $A_{1}$, obtaining $A_{2}$

3. Either leave the matrix $A_{2}$ as it stands or take its transpose, obtaining $A_{3}=B$.

Obviously, this is an equivalence relation and it is clear that these three operations are independent. This equivalence relation partitions $\mathbf{M}$ into equivalence classes. The number of distinct problems of permutations on $n$ objects with restricted position is thus the number of equivalence classes of the matrices in $\mathbf{M}$. In the above quotation from Riordan [4], the presence of an $x$ in his matrix corresponds to that of a 1 in the associated binary matrix, while a blank space in his matrix becomes a 0 in the binary matrix.

A graph consists of a finite collection of points together with lines joining certain pairs of distinct points. When two points are joined by a line, they are adjacent. A graph is said to be colored with $k$ colors if each point is assigned one of these colors, any two adjacent points have different colors, and all $k$ colors are used. A bicolored graph is one which has been colored with two colors.

To a given restricted permutation problem represented by the binary matrix $A=\left(a_{i j}\right)$, there corresponds the bicolored graph with $2 n$ points $1,2, \cdots, n, 1^{\prime}$, $2^{\prime}, \cdots, n^{\prime}$ in which point $i$ is joined by a line to point $j^{\prime}$ if and only if $a_{i j}=1$. Thus for $n=4$, the rencontres and reduced ménage problems give the bicolored graphs of Figure 1. 

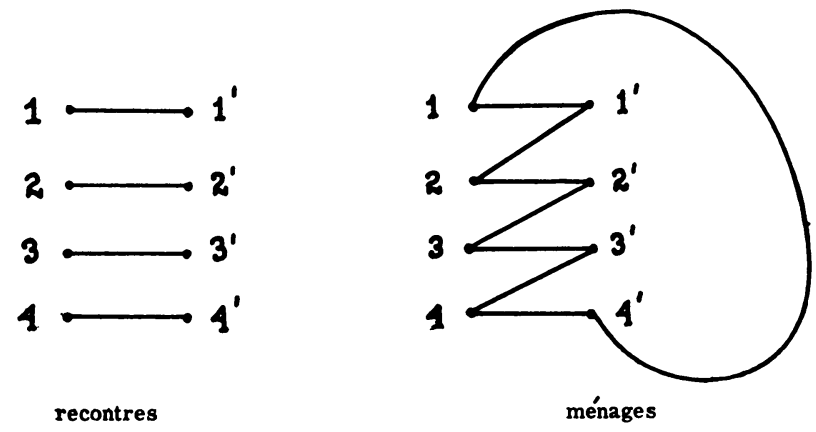

Fig. 1

Two graphs are isomorphic if there is a one-to-one correspondence between their set of points which preserves adjacency. Two bicolored graphs are chromatically isomorphic if there is an isomorphism between them which preserves color, i.e., two points of the first graph have different colors if and only if their image points do. Clearly, chromatic isomorphism is an equivalence relation on bicolored graphs. As an illustration, we show in Figure 2 all bicolored graphs (up to chromatic isomorphism) in which there are two points of each color, together with the corresponding matrices.

LEMma. Two square binary matrices are equivalent if and only if the corresponding bicolored graphs are chromatically isomorphic.

Proof. We translate the three defining operations for equivalence of matrices into graphical terms. Any permutation of the rows of a matrix $A$ in $\mathbf{M}$ corresponds to a renumbering of the $n$ points of the first color in the associated bicolored graph $G$. A permutation of the columns of $A$ becomes a renumbering of the $n$ points of the second color. Finally, transposing the matrix $A$ amounts to interchanging the two colors assigned to the points of $G$. Clearly, these three operations serve to characterize chromatic isomorphism.

A formula for the counting polynomial

$$
g_{n n}(x)=\sum_{q=0}^{n^{2}} b_{q} x^{q}
$$

where $b_{q}$ is the number of chromatically nonisomorphic bicolored graphs with $n$ points of each color and $q$ lines which have been found in [2]. Let $P_{n}$ be the number of inequivalent matrices in $\mathbf{M}$, i.e., the number of distinct types of restricted permutation problems (on $n$ objects); then

$$
P_{n}=g_{n n}(1)=b_{0}+b_{\mathrm{i}}+\cdots+b_{n^{2}} .
$$

For example, we see from Figure 2 that $g_{22}(x)=1+x+2 x^{2}+x^{3}+x^{4}$; hence $P_{2}=6$. The number $P_{n}$ may be found from the cycle index of the "exponentiation group" $S_{n}^{s_{2}}$ (where $S_{n}$ is the symmetric group of degree $n$ ) using the enumeration lemma of [1]. This is the same procedure as substituting 1 for $x$ in the formula for $g_{n n}(x)$, which is derived using Pólya's method [3]. To give the result conveniently, we require the following notation: 
$(i)=\left(i_{1}, i_{2}, \cdots, i_{n}\right)$ denotes a partition of $n$ such that:

$$
\begin{gathered}
i_{1}+2 i_{2}+\cdots+n i_{n}=n, \\
\nu(i)=\prod_{k=1}^{n} k^{i_{k}} i_{k} !
\end{gathered}
$$

and $d(r, s)$ is the greatest common divisor of the positive integers $r$ and $s$.

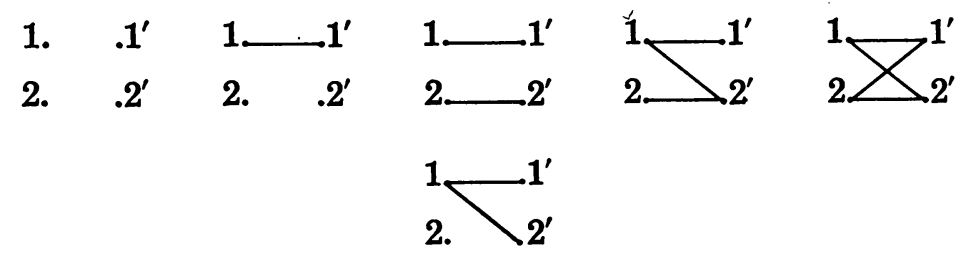

$$
\begin{aligned}
{\left[\begin{array}{ll}
0 & 0 \\
0 & 0
\end{array}\right]\left[\begin{array}{ll}
1 & 0 \\
0 & 0
\end{array}\right] } & {\left[\begin{array}{ll}
1 & 0 \\
0 & 1
\end{array}\right] } \\
& {\left[\begin{array}{ll}
1 & 1 \\
0 & 0
\end{array}\right] }
\end{aligned}
$$

Fig. 2

We may now state the formula for $P_{n}$. Let

$$
\begin{gathered}
\alpha(i, j)=\sum_{r, s=1}^{n} i_{r} j_{s} d(r, s) \\
\beta(j)=\sum_{k \text { even }} k\left[\frac{j_{k}}{2}+\left(\begin{array}{c}
j_{k} \\
2
\end{array}\right)\right]+\sum_{k \text { odd }}\left[(k+1) \frac{j_{k}}{2}+k\left(\begin{array}{c}
j_{k} \\
2
\end{array}\right)\right]+\sum_{r<s} j_{r} j_{s} d(r, s) .
\end{gathered}
$$

Then

$$
P_{n}=\frac{1}{2} \sum_{(i),(j)} \frac{1}{\nu(i) \nu(j)} 2^{\alpha(i, j)}+\frac{1}{2} \sum_{(j)} \frac{1}{\nu(j)} 2^{\beta(j)}
$$

where the first sum is taken over all pairs $(i),(j)$ of partitions of $n$ and the second sum is over all partitions $(j)$ of $n$.

We illustrate for $n=3$ whose three partitions $\pi_{1}, \pi_{2}, \pi_{3}$ are $1+1+1,1+2$, and 3 . These may be written as the sequences

$$
(3,0,0), \quad(1,1,0), \quad(0,0,1)
$$

respectively. The values of $\alpha\left(\pi_{i}, \pi_{j}\right)$ for $n=3$ are given in the matrix:

$\alpha\left(\pi_{i}, \pi_{j}\right)$

$$
i\left[\begin{array}{lll}
9 & 6 & 3 \\
6 & 5 & 2 \\
3 & 2 & 3
\end{array}\right]
$$


while the values of $\nu\left(\pi_{i}\right)$ and $\beta\left(\pi_{i}\right)$ are given in the table:

\begin{tabular}{ccc}
$i$ & $\nu\left(\pi_{i}\right)$ & $\beta\left(\pi_{i}\right)$ \\
\hline 1 & 6 & 6 \\
2 & 2 & 3 \\
3 & 3 & 2
\end{tabular}

Hence we have

$$
P_{3}=\frac{1}{2}\left[\frac{2^{9}}{6^{2}}+\frac{2^{5}}{2^{2}}+\frac{2^{3}}{3^{2}}+\frac{2^{7}}{12}+\frac{2^{4}}{18}+\frac{2^{3}}{6}\right]+\frac{1}{2}\left[\frac{2^{6}}{6}+\frac{2^{3}}{2}+\frac{2^{2}}{3}\right]=26
$$

University of Michigan

Ann Arbor, Michigan

1. F. HARARY, "Note on an enumeration theorem of Davis and Slepian," Michigan Math. $J$., v. 3, 1955-56, p. 149-153.

2. F. HARARY, "On the number of bicolored graphs," Pacific J. Math., v. 8, 1958, p. 743-755.

3. G. P6LYA, "Kombinatorische Anazahlbestimmungen für Gruppen, Graphen und chemische Verbindungen," Acta Math., v. 68, 1937, p. 145-254.

4. J. RIORDAN, An Introduction to Combinatorial Analysis, Wiley \& Sons, New York, 1958

\section{Evaluation of the Zeros of Cross-Product Bessel Functions}

\section{By L. Jackson Laslett and William Lewish}

1. Introduction. There is considerable interest in the zeros of certain crossproduct Bessel functions which arise in solving Bessel's equation subject to Dirichlet or Neumann boundary conditions at $r=a, b$,

$$
J_{n}(q a) Y_{n}(q b)-J_{n}(q b) Y_{n}(q a)=0
$$

or

$$
J_{n}^{\prime}(q a) Y_{n}^{\prime}(q b)-J_{n}^{\prime}(q b) Y_{n}^{\prime}(q a)=0,
$$

because of their well-known application in physical or engineering problems for which the use of cylindrical coordinates is appropriate. In many instances attention may be directed primarily to the zeros of such functions when $n$ is not large because of the interest in the lower-order modes which are possible in the physical problem under consideration, but cases may also arise in which the higher-order modes will warrant attention in order to determine the circumstances in which such possibly unwanted modes may become excited.

Solutions to (1a) and (1b) have been discussed by a number of writers [1], [6], and results presented in the form of algebraic formulas, in tables, or graphically. For application to problems in which $(b-a) /(b+a)$ is small and in which $n$ may

Received February 1, 1961. The work reported in this paper was supported by the U. S. Atomic Energy Commission. It is a pleasure also to acknowledge the assistance provided by Dr. C. L. Hammer in discussing with us the use of the perturbation method for which results are reported in Section 3. 